\title{
Oscillation of Second-Order Neutral Differential Equations
}

\author{
By
}

\author{
Tongxing $\mathrm{LI}^{1}$, Yuri V. Rogovchenko ${ }^{2}$ and Chenghui ZHANG ${ }^{3}$ \\ (Shandong University ${ }^{1,3}$, P. R. China, and University of $\operatorname{Agder}^{2}$, Norway)
}

\begin{abstract}
We study oscillatory behavior of a class of second-order neutral differential equations relating oscillation of these equations to existence of positive solutions to associated first-order functional differential inequalities. Our assumptions allow applications to differential equations with both delayed and advanced arguments, and not only. New theorems complement and improve a number of results reported in the literature. Two illustrative examples are provided.

Key Words and Phrases. Oscillation, Neutral differential equations, Delayed arguments, Advanced arguments, Positive solutions, Comparison.

2010 Mathematics Subject Classification Numbers. 34K11.
\end{abstract}

\section{Introduction}

In this paper, we study the oscillation of a class of functional differential equations

$$
\left(r(t)[x(t)+p(t) x(\tau(t))]^{\prime}\right)^{\prime}+q(t) x(\sigma(t))=0
$$

in the case

$$
\lim _{t \rightarrow \infty} R(t)<\infty,
$$

where $R(t)=\int_{t_{0}}^{t}(r(s))^{-1} \mathrm{~d} s$. The increasing interest in oscillatory properties of solutions to second-order neutral differential equations is motivated by their applications in the engineering and natural sciences. We refer the reader to the papers [1]-[15] and the references cited therein.

We assume that the following assumptions are satisfied:

$$
\begin{aligned}
& \left(H_{1}\right) \quad r, p, q \in \mathrm{C}\left(\left[t_{0}, \infty\right)\right), r(t)>0,0 \leq p(t) \leq p_{0}<\infty, q(t)>0 ; \\
& \left(H_{2}\right) \quad \sigma \in \mathrm{C}^{1}\left(\left[t_{0}, \infty\right)\right), \lim _{t \rightarrow \infty} \sigma(t)=\infty ; \\
& \left(H_{3}\right) \quad \tau \in \mathrm{C}^{1}\left(\left[t_{0}, \infty\right)\right), \tau^{\prime}(t) \geq \tau_{0}>0, \tau \circ \sigma=\sigma \circ \tau .
\end{aligned}
$$

Throughout the paper, we use the notation $z(t):=x(t)+p(t) x(\tau(t))$. By a solution of equation (1), we mean a function $x \in \mathrm{C}\left(\left[T_{x}, \infty\right)\right), T_{x} \geq t_{0}$, such that $r z^{\prime} \in \mathrm{C}^{1}\left(\left[T_{x}, \infty\right)\right)$ and $x$ satisfies $(1)$ on $\left[T_{x}, \infty\right)$. We consider only those solutions of (1) which satisfy $\sup \{|x(t)|: t \geq T\}>0$ for all $T \geq T_{x}$ and assume that (1) possesses such solutions. A solution of (1) is called oscillatory if it does 
not have the largest zero on $\left[T_{x}, \infty\right)$, otherwise, it is called non-oscillatory. Equation (1) is said to be oscillatory if all its solutions are oscillatory.

Very recently, Baculíková and Džurina [4] established several oscillation theorems for equation (1) via its comparison with associated first-order delay differential equations in the case when, in addition to $\left(H_{1}\right)-\left(H_{3}\right)$, condition

$$
\lim _{t \rightarrow \infty} R(t)=\infty
$$

is satisfied. Assuming

$$
\int_{t_{0}}^{\infty} r^{-1 / \gamma}(t) \mathrm{d} t=\infty
$$

instead of (3), Baculíková and Džurina [5] extended results of [4] to a nonlinear neutral differential equation

$$
\left(r(t)\left[(x(t)+p(t) x(\tau(t)))^{\prime}\right]^{\gamma}\right)^{\prime}+q(t) x^{\beta}(\sigma(t))=0,
$$

where $\gamma$ and $\beta$ are ratios of positive odd integers. Using a similar idea, Baculíková et al. [6] studied the oscillation of an even-order neutral differential equation

$$
\left(\left[(x(t)+p(t) x(\tau(t)))^{(n-1)}\right]^{\gamma}\right)^{\prime}+q(t) x^{\gamma}(\sigma(t))=0,
$$

where $n \geq 2$ is an even number and $\gamma \geq 1$ is a ratio of two odd positive integers. Han et al. [10] employed Riccati transformation and integral averaging techniques to investigate the oscillation of a nonlinear differential equation

$$
\left(r(t) \psi(x(t))\left[(x(t)+p(t) x(\tau(t)))^{\prime}\right]^{\gamma}\right)^{\prime}+q(t) f(x(\sigma(t)))=0
$$

in the case when $\left(H_{1}\right),\left(H_{2}\right)$ and $\int_{t_{0}}^{\infty} r^{-1 / \gamma}(t) \mathrm{d} t<\infty$ hold, $\tau(t) \leq t, \sigma(t) \leq t$, $\sigma^{\prime}(t)>0$, and

$$
\text { either } \quad \sigma(t) \geq \tau(t), \quad \text { or } \quad \sigma(t) \leq \tau(t) .
$$

Li et al. [14] studied oscillation of equation (1) under the assumptions that (2) holds, $\tau$ and $\sigma$ are strictly increasing, $p(t)>1$, and (4) is satisfied. Li et al. [15] analyzed oscillatory properties of equation (1) in the case where $\left(H_{1}\right)-\left(H_{3}\right)$ hold, $\tau(t) \geq t$, and $\sigma(t) \geq t$. They derived a sufficient condition which ensures that solutions of equation (1) are either oscillatory or satisfy $\lim _{t \rightarrow \infty} x(t)=0$ for the case where (2) holds and $0 \leq p(t) \leq p_{1}<1$ [15, Theorem 3.8]. Finally, we note that Hasanbulli and Rogovchenko [11] obtained new oscillation results for a nonlinear neutral differential equation

$$
\left(r(t)[x(t)+p(t) x(t-\tau)]^{\prime}\right)^{\prime}+q(t) f(x(t), x(\sigma(t)))=0
$$

in the case where $0 \leq p(t) \leq 1, \sigma(t) \leq t, \sigma^{\prime}(t)>0$, and (3) holds. 
We stress that theorems in $[4,5,6,11]$ cannot be applied to (1) in the case where (2) holds. The purpose of this paper is to extend a new method for the analysis of the oscillation of equation (1) via comparison principles suggested by Baculíková and Džurina [4] for the study of (1) under the assumption (2).

\section{Main results}

In what follows, all inequalities are assumed to hold eventually, that is, for all $t$ large enough. We also use the notation $y(t):=-u(t):=r(t) z^{\prime}(t), Q(t):=$ $\min \{q(t), q(\tau(t))\}$, and $\delta(t):=\int_{t}^{\infty}(r(s))^{-1} \mathrm{~d} s$.

Theorem 1. Assume that $\left(H_{1}\right)-\left(H_{3}\right)$ and (2) hold, and let $t_{1}$ be large enough. Suppose that there exist two functions $\eta, \xi \in \mathrm{C}\left(\left[t_{0}, \infty\right)\right)$ such that $\eta(t) \leq \sigma(t) \leq \xi(t)$ and $\lim _{t \rightarrow \infty} \eta(t)=\infty$. If the first-order neutral differential inequalities

$$
\left(y(t)+\frac{p_{0}}{\tau_{0}} y(\tau(t))\right)^{\prime}+Q(t)\left(R(\eta(t))-R\left(t_{1}\right)\right) y(\eta(t)) \leq 0
$$

and

$$
\left(w(t)+\frac{p_{0}}{\tau_{0}} w(\tau(t))\right)^{\prime}-Q(t) \delta(\xi(t)) w(\xi(t)) \geq 0
$$

have no positive solutions, equation (1) is oscillatory.

Proof. Assume that (1) has a non-oscillatory solution $x(t)$ on $\left[t_{0}, \infty\right)$. Without loss of generality, we can suppose that $x(t)$ is eventually positive. As in the proof of $[4$, Theorem 1], one arrives at the inequality

$$
\left(r(t) z^{\prime}(t)\right)^{\prime}+\frac{p_{0}}{\tau_{0}}\left(r(\tau(t)) z^{\prime}(\tau(t))\right)^{\prime}+Q(t) z(\sigma(t)) \leq 0 .
$$

Equation (1) yields that, for some $t_{1}$ large enough and for all $t \geq t_{1}$, either

$$
z^{\prime}(t)>0, \quad\left(r(t) z^{\prime}(t)\right)^{\prime}<0
$$

or

$$
z^{\prime}(t)<0, \quad\left(r(t) z^{\prime}(t)\right)^{\prime}<0
$$

Assume first that (8) holds. Inequality (7) and the fact that $\eta(t) \leq \sigma(t)$ yield

$$
\left(r(t) z^{\prime}(t)\right)^{\prime}+\frac{p_{0}}{\tau_{0}}\left(r(\tau(t)) z^{\prime}(\tau(t))\right)^{\prime}+Q(t) z(\eta(t)) \leq 0 .
$$


It follows from condition (8) that

$$
z(t) \geq \int_{t_{1}}^{t} \frac{r(s) z^{\prime}(s)}{r(s)} \mathrm{d} s \geq r(t) z^{\prime}(t) \int_{t_{1}}^{t} \frac{1}{r(s)} \mathrm{d} s,
$$

for all sufficiently large $t$. Therefore, $y(t)$ is a positive solution of inequality (5), which contradicts our assumption that this inequality has no positive solutions.

Consider now the second case. It follows from (9) that

$$
z^{\prime}(s) \leq \frac{r(t) z^{\prime}(t)}{r(s)} \quad \text { for all } s \geq t
$$

which, upon integration, leads to

$$
z(l) \leq z(t)+r(t) z^{\prime}(t) \int_{t}^{l} \frac{1}{r(s)} \mathrm{d} s .
$$

Passing to the limit as $l \rightarrow \infty$, we conclude that

$$
z(t)+r(t) z^{\prime}(t) \int_{t}^{\infty} \frac{1}{r(s)} \mathrm{d} s \geq 0 .
$$

Therefore,

$$
z(t) \geq-r(t) z^{\prime}(t) \delta(t) .
$$

It follows from (7) and condition $\sigma(t) \leq \xi(t)$ that

$$
\left(r(t) z^{\prime}(t)\right)^{\prime}+\frac{p_{0}}{\tau_{0}}\left(r(\tau(t)) z^{\prime}(\tau(t))\right)^{\prime}+Q(t) z(\xi(t)) \leq 0 .
$$

Then, $y(t)<0$ and, by virtue of (10) and (11),

$$
\left(y(t)+\frac{p_{0}}{\tau_{0}} y(\tau(t))\right)^{\prime}-Q(t) \delta(\xi(t)) y(\xi(t)) \leq 0 .
$$

Writing the latter inequality in the form

$$
-\left(y(t)+\frac{p_{0}}{\tau_{0}} y(\tau(t))\right)^{\prime}-Q(t) \delta(\xi(t))(-y(\xi(t))) \geq 0,
$$

we deduce that $u(t)$ is a positive solution of the inequality (6), which, according to our assumption, has no positive solutions. Therefore, equation (1) is oscillatory.

Under additional conditions on the coefficients of (1), one can deduce from Theorem 1 a number of oscillation criteria applicable to different classes of 
equations. In what follows, the notation $\tau^{-1}$ stands for the inverse of the function $\tau$.

Theorem 2. Assume that $\left(H_{1}\right)-\left(H_{3}\right)$ and (2) hold, and let $t_{1}$ be large enough. Suppose that there exist two functions $\eta, \xi \in \mathrm{C}\left(\left[t_{0}, \infty\right)\right)$ such that $\eta(t) \leq \sigma(t) \leq \xi(t)$ and $\lim _{t \rightarrow \infty} \eta(t)=\infty$. Assume also that

$$
\tau(t) \geq t
$$

If the first-order functional differential inequalities

$$
h^{\prime}(t)+\frac{\tau_{0}}{\tau_{0}+p_{0}} Q(t)\left(R(\eta(t))-R\left(t_{1}\right)\right) h(\eta(t)) \leq 0
$$

and

$$
f^{\prime}(t)-\frac{\tau_{0}}{\tau_{0}+p_{0}} Q(t) \delta(\xi(t)) f\left(\tau^{-1}(\xi(t))\right) \geq 0
$$

have no positive solutions, equation (1) is oscillatory.

Proof. Suppose that (1) has a non-oscillatory solution $x(t)$ defined on $\left[t_{0}, \infty\right)$. As above, we can assume that $x(t)$ is eventually positive. Following the same lines as in [4, Theorem 2], we conclude that the inequality (13) has a positive solution, which contradicts our assumption. On the other hand, we have shown in Theorem 1 that the function $u(t)$ is positive, increasing, and satisfies (6). It follows from (12) that

$$
w(t) \leq u(\tau(t))\left(1+\frac{p_{0}}{\tau_{0}}\right)
$$

where

$$
w(t):=u(t)+\frac{p_{0}}{\tau_{0}} u(\tau(t))
$$

Substituting (15) into (6), we conclude that $w(t)$ is a positive solution of (14), which contradicts the fact that this inequality does not have positive solutions. The proof is complete.

Combining Theorem 2 with the oscillation criteria presented in Ladde et al. [12, Theorems 2.1.1 and 2.4.1], we obtain the following result.

Corollary 3. Assume that conditions $\left(H_{1}\right)-\left(H_{3}\right),(2)$ and (12) are satisfied. Suppose further that there exist two functions $\eta, \xi \in \mathrm{C}\left(\left[t_{0}, \infty\right)\right)$ such that $\eta(t)<t$, $\xi(t)>\tau(t), \eta(t) \leq \sigma(t) \leq \xi(t)$, and $\lim _{t \rightarrow \infty} \eta(t)=\infty$. If, for all sufficiently large 
$t_{1} \geq t_{0}$

$$
\liminf _{t \rightarrow \infty} \int_{\eta(t)}^{t} Q(s)\left(R(\eta(s))-R\left(t_{1}\right)\right) \mathrm{d} s>\frac{\tau_{0}+p_{0}}{\tau_{0} \mathrm{e}}
$$

and

$$
\liminf _{t \rightarrow \infty} \int_{t}^{\tau^{-1}(\xi(t))} Q(s) \delta(\xi(s)) \mathrm{d} s>\frac{\tau_{0}+p_{0}}{\tau_{0} \mathrm{e}},
$$

equation (1) is oscillatory.

Proof. By [12, Theorem 2.1.1], assumption (17) ensures that the differential inequality (13) has no positive solutions. On the other hand, by [12, Theorem 2.4.1], condition (18) guarantees that the differential inequality (14) has no positive solutions. Application of Theorem 2 yields the result.

Theorem 4. Assume that conditions $\left(H_{1}\right)-\left(H_{3}\right)$ and (2) are satisfied, and let $t_{1}$ be large enough. Suppose that there exist two functions $\eta, \xi \in \mathrm{C}\left(\left[t_{0}, \infty\right)\right)$ such that $\eta(t) \leq \sigma(t) \leq \xi(t)$ and $\lim _{t \rightarrow \infty} \eta(t)=\infty$. Finally, assume that

$$
\tau(t) \leq t .
$$

If the first-order functional differential inequalities

$$
h^{\prime}(t)+\frac{\tau_{0}}{\tau_{0}+p_{0}} Q(t)\left(R(\eta(t))-R\left(t_{1}\right)\right) h\left(\tau^{-1}(\eta(t))\right) \leq 0
$$

and

$$
f^{\prime}(t)-\frac{\tau_{0}}{\tau_{0}+p_{0}} Q(t) \delta(\xi(t)) f(\xi(t)) \geq 0
$$

have no positive solutions, equation (1) is oscillatory.

Proof. Assume again that equation (1) has on $\left[t_{0}, \infty\right)$ a non-oscillatory solution $x(t)$ which is eventually positive. Along the same lines as in [4, Theorem 3], we deduce that the inequality (20) has a positive solution, which contradicts our assumption. On the other hand, it has been established in Theorem 1 that the function $y(t)$ is positive, increasing, and satisfies the inequality (6). By virtue of (19), the inequality

$$
w(t) \leq u(t)\left(1+\frac{p_{0}}{\tau_{0}}\right)
$$

holds for the function $w(t)$ defined by (16). Substituting this into (6), we conclude that $w(t)$ is a positive solution of (21), which contradicts our assumption. The proof is complete. 
Combining Theorem 4 with results in Ladde et al. [12, Theorems 2.1.1 and 2.4.1], we obtain the following oscillation criterion.

Corollary 5. Assume that conditions $\left(H_{1}\right)-\left(H_{3}\right),(2)$, and (19) are satisfied. Suppose further that there exist two functions $\eta, \xi \in \mathrm{C}\left(\left[t_{0}, \infty\right)\right)$ such that $\eta(t)<\tau(t), \xi(t)>t, \eta(t) \leq \sigma(t) \leq \xi(t)$, and $\lim _{t \rightarrow \infty} \eta(t)=\infty$. If, for all sufficiently large $t_{1} \geq t_{0}$,

$$
\liminf _{t \rightarrow \infty} \int_{\tau^{-1}(\eta(t))}^{t} Q(s)\left(R(\eta(s))-R\left(t_{1}\right)\right) \mathrm{d} s>\frac{\tau_{0}+p_{0}}{\tau_{0} \mathrm{e}}
$$

and

$$
\liminf _{t \rightarrow \infty} \int_{t}^{\xi(t)} Q(s) \delta(\xi(s)) \mathrm{d} s>\frac{\tau_{0}+p_{0}}{\tau_{0} \mathrm{e}},
$$

equation (1) is oscillatory.

Proof. By virtue of [12, Theorem 2.1.1], condition (22) ensures that differential inequality (20) has no positive solutions. On the other hand, it follows from [12, Theorem 2.4.1] that condition (23) guarantees that the differential inequality (21) has no positive solutions. Application of Theorem 4 completes the proof.

\section{Examples and discussion}

The following two examples illustrate applications of theoretical results in the previous section.

Example 6. For $t \geq 1$, consider a second-order neutral differential equation

$$
\left(t^{2}\left[x(t)+p_{0} x(\alpha t)\right]^{\prime}\right)^{\prime}+q_{0} x(\beta t)=0,
$$

where $\alpha, p_{0}, q_{0}$, and $\beta$ are positive constants, $\beta>\alpha>1$. Let $\eta(t)=t / \alpha$ and $\xi(t)=\beta t$. Application of Corollary 3 yields that all solutions of equation (24) are oscillatory provided that

$$
\frac{q_{0}}{\beta} \ln \frac{\beta}{\alpha}>\frac{\alpha+p_{0}}{\alpha \mathrm{e}}
$$

Example 7. For $t \geq 1$, consider a second-order neutral delay differential equation

$$
\left(\mathrm{e}^{t}\left[x(t)+\frac{1}{2} x\left(t-\frac{\pi}{4}\right)\right]^{\prime}\right)^{\prime}+12 \sqrt{65} \mathrm{e}^{t} x\left(t-\frac{1}{8} \arcsin \frac{\sqrt{65}}{65}\right)=0 .
$$


Let $\eta(t)=t-\pi / 2$ and $\xi(t)=t+\pi / 4$. Taking into account that $R(t)=$ $\mathrm{e}^{-1}-\mathrm{e}^{-t}, Q(t)=12 \sqrt{65} \mathrm{e}^{t-\pi / 4}, \delta(t)=\mathrm{e}^{-t}$, and $\tau_{0}=1$, we conclude that

$$
\begin{aligned}
& \liminf _{t \rightarrow \infty} \int_{\tau^{-1}(\eta(t))}^{t} Q(s)\left(R(\eta(s))-R\left(t_{1}\right)\right) \mathrm{d} s \\
& \quad=\liminf _{t \rightarrow \infty} \int_{t-\pi / 4}^{t} 12 \sqrt{65} \mathrm{e}^{s-\pi / 4}\left(\mathrm{e}^{-t_{1}}-\mathrm{e}^{-s+\pi / 2}\right) \mathrm{d} s>\frac{3}{2 \mathrm{e}}
\end{aligned}
$$

and

$$
\liminf _{t \rightarrow \infty} \int_{t}^{\xi(t)} Q(s) \delta(\xi(s)) \mathrm{d} s=\liminf _{t \rightarrow \infty} \int_{t}^{t+\pi / 4} 12 \sqrt{65} \mathrm{e}^{s-\pi / 4} \mathrm{e}^{-s-\pi / 4} \mathrm{~d} s>\frac{3}{2 \mathrm{e}} .
$$

By Corollary 5, all solutions to equation (25) oscillate. As a matter of fact, one such solution is $x(t)=\sin 8 t$.

Remark 8. Oscillation criteria established in this paper for equation (1) complement, on one hand, theorems reported by Baculíková and Džurina [4] and Hasanbulli and Rogovchenko [11] because we use assumption (2) rather than (3) and, on the other hand, those by Li et al. [14] since our criteria can be applied to the case where $0 \leq p(t) \leq p_{0}<\infty$.

Remark 9. Using methods different from those exploited in $[10,11,14$, 15], we improve results of Han et al. [10] and Li et al. [15, Theorem 3.8] by removing assumptions $\sigma(t) \leq \tau(t) \leq t$ or $t \geq \sigma(t) \geq \tau(t)$ imposed in the cited papers and by providing sufficient conditions which ensure that all solutions of (1) are oscillatory.

Remark 10. As mentioned by Baculíková and Džurina [4, Remark 4], assumptions $\left(\mathrm{H}_{2}\right)$ and $\left(\mathrm{H}_{3}\right)$ on functional arguments do not specify whether $\tau(t)$ is a delayed or advanced argument. Furthermore, $\sigma(t)$ can be a delayed argument and $\sigma(t)-t$ can even oscillate. However, to achieve such flexibility, we are forced to require, as in [4], monotonicity of $\tau$ and that $\tau \circ \sigma=\sigma \circ \tau$. The question regarding the study of oscillatory properties of equation (1) with other methods that do not require assumption $\left(H_{3}\right)$ remains open at the moment.

Remark 11. Note that by using the inequalities

$$
x_{1}^{\alpha}+x_{2}^{\alpha} \geq\left(x_{1}+x_{2}\right)^{\alpha}, \quad \text { for } 0<\alpha \leq 1 \text { and all } x_{1}, x_{2} \in[0, \infty),
$$

and

$$
x_{1}^{\alpha}+x_{2}^{\alpha} \geq \frac{1}{2^{\alpha-1}}\left(x_{1}+x_{2}\right)^{\alpha}, \quad \text { for } \alpha \geq 1 \text { and all } x_{1}, x_{2} \in[0, \infty) \text {, }
$$


results reported in this paper can be extended to a second-order half-linear neutral differential equation

$$
\left(r(t)\left([x(t)+p(t) x(\tau(t))]^{\prime}\right)^{\alpha}\right)^{\prime}+q(t) x^{\alpha}(\sigma(t))=0,
$$

where $\alpha>0$ is a ratio of odd positive integers.

Acknowledgement. The research of the first and the third authors was supported by NNSF of P.R. China (Grant No. 61034007, 51277116, 50977054). The first author would like to express his gratitude to Professors Ravi P. Agarwal and Martin Bohner for their interest in this work and useful comments. The authors thank the anonymous referee for careful reading of the manuscript and pointing out an inaccuracy in our example.

\section{References}

[1] Agarwal, Ravi P. and Grace, Said R., Oscillation theorems for certain neutral functional differential equations, Comput. Math. Appl., 38 (1999), 1-11.

[2 ] Agarwal, Ravi P., Grace, Said R. and O'Regan, Donal, Oscillation Theory for Difference and Functional Differential Equations, Kluwer Academic Publishers, Dordrecht, 2000.

[ 3 ] Agarwal, Ravi P., Grace, Said R. and O'Regan, Donal, Oscillation criteria for certain $n$-th order differential equations with deviating arguments, J. Math. Anal. Appl., 262 (2001), 601622.

[4] Baculíková, Blanka and Džurina, Jozef, Oscillation theorems for second order neutral differential equations, Comput. Math. Appl., 61 (2011), 94-99.

[5] Baculíková, Blanka and Džurina, Jozef, Oscillation theorems for second order nonlinear neutral differential equations, Comput. Math. Appl., 62 (2011), 4472-4478.

[6] Baculíková, Blanka, Džurina, Jozef and Li, Tongxing, Oscillation results for even-order quasilinear neutral functional differential equations, Electron. J. Differential Equations, 2011 (2011), No. 143, 9pp.

[7] Erbe, Lynn, Kong, Qingkai and Zhang, Binggen, Oscillation Theory for Functional Differential Equations, Monographs and Textbooks in Pure and Applied Mathematics, 190, Marcel Dekker, Inc., New York, 1995.

[8] Grace, Said R. and Lalli, B. S., Oscillation of nonlinear second order neutral delay differential equations, Rad. Mat., 3 (1987), 77-84.

[9] Grammatikopoulos, M. K., Ladas, G. and Meimaridou, A., Oscillation of second order neutral delay differential equation, Rad. Mat., 1 (1985), 267-274.

[10] Han, Zhenlai, Li, Tongxing, Sun, Shurong and Sun, Yibing, Remarks on the paper [Appl. Math. Comput. 207 (2009) 388-396], Appl. Math. Comput., 215 (2010), 3998-4007.

[11] Hasanbulli, Mustafa and Rogovchenko, Yuri V., Oscillation criteria for second order nonlinear neutral differential equations, Appl. Math. Comput., 215 (2010), 4392-4399.

[12] Ladde, G. S., Lakshmikantham, V. and Zhang, B. G., Oscillation Theory of Differential Equations with Deviating Arguments, Monographs and Textbooks in Pure and Applied Mathematics, 110, Marcel Dekker, Inc., New York, 1987.

[13] Li, Tongxing, Comparison theorems for second-order neutral differential equations of mixed type, Electron. J. Differential Equations, 2010 (2010), No. 167, 7pp. 
[14] Li, Tongxing, Agarwal, Ravi P. and Bohner, Martin, Some oscillation results for secondorder neutral differential equations, J. Indian Math. Soc. (N.S.), 79 (2012), 97-106.

[15] Li, Tongxing, Han, Zhenlai, Zhang, Chenghui and Li, Hua, Oscillation criteria for secondorder superlinear neutral differential equations, Abstr. Appl. Anal., 2011 (2011), Art. ID $367541,17 \mathrm{pp}$.

\author{
nuna adreso: \\ Tongxing $\mathrm{Li}$ \\ School of Control Science and Engineering \\ Shandong University \\ Jinan, Shandong 250061 \\ P. R. China \\ E-mail: litongx2007@163.com \\ Yuri V. Rogovchenko \\ Faculty of Engineering and Science \\ University of Agder \\ 422, N-4604, Kristiansand \\ Norway \\ E-mail: yuriy.rogovchenko@uia.no \\ Chenghui Zhang \\ School of Control Science and Engineering \\ Shandong University \\ Jinan, Shandong 250061 \\ P. R. China \\ E-mail: zchui@sdu.edu.cn
}

(Ricevita la 22-an de marto, 2012)

(Reviziita la 18-an de majo, 2012) 